\title{
Risperidone Induced Pisa Syndrome in a Male Adolescent
}

\author{
Serkan Güneş ${ }^{1}$, Özalp Ekinci', Meltem Çobanoğulları Direk ${ }^{2}$, Veli Yıldırım, Çetin Okuyaz ${ }^{2}$, Fevziye Toros ${ }^{1}$ \\ ${ }^{1}$ Department of Child and Adolescent Psychiatry, ${ }^{2}$ Department of Pediatric Neurology, Mersin University Medical Faculty, Mersin, Turkey
}

\begin{abstract}
Pisa syndrome, a rare dystonic reaction resulting from prolonged exposure to antipsychotic medications, is characterized by persistent dystonia of trunk muscles and abnormal posture. It is called Pisa syndrome, because the abnormal posture resembles the Leaning Tower of Pisa. Different from other types of dystonic reactions, Pisa syndrome is more prevalent in females and in older patients with organic brain changes. A 15-year-old male patient with mental retardation was admitted to pediatric neurology clinic for the complaint of abnormal posturing. He had been taking only risperidone for the last four years. Over the last month, the patient gradually developed tonic flexion of trunk and head toward left and was diagnosed with Pisa syndrome. In this paper, we aimed to discuss the association between risperidone use and Pisa syndrome in light of the available literature.
\end{abstract}

KEY WORDS: Pisa syndrome; Adolescent; Child; Case reports; Risperidone.

\section{INTRODUCTION}

Risperidone is an atypical antipsychotic with high affinity at dopamine $\mathrm{D}_{2}$ and serotonin $5 \mathrm{HT}_{2}$ receptors. In children and adolescent, risperidone is used for schizophrenia, conduct disorder, bipolar disorder, tic disorder, obsessive-compulsive disorder and behavioral problems accompanied with autistic disorder and mental retardation. Risperidone, although rarely, may cause extrapyramidal side effects such as parkinsonism, akathisia, dyskinesia or dystonia. ${ }^{1,2)}$

Dystonia is a movement disorder that manifests with involuntary and persistent muscle spasms involving one or more body parts. Pisa syndrome (PS) is a rare form of acute or tardive dystonia. ${ }^{3)}$ The incidence of antipsychoticinduced PS is reported as $0.04 \%$. Although it frequently results from prolonged exposure to typical antipsychotics, PS may also occur with atypical antipsychotics and other medications including tricyclic antidepressants, antiemetics and cholinesterase inhibitors. ${ }^{4-7)}$

PS is characterized by a persistent dystonia of trunk muscles from cervical to lumbar region, tonic lateral flexion of the trunk together with slightly axial rotation and

\footnotetext{
Received: June 5, 2015/Revised: July 9, 2015

Accepted: July 24, 2015

Address for correspondence: Serkan Güneş, MD

Department of Child and Adolescent Psychiatry, Mersin University

Medical Faculty, Çiftlikköy Kampüsü, Yenişehir, Mersin, Turkey

Tel: +90-5542587368, Fax: +90-3242410092

E-mail: dr_sgunes@hotmail.com
}

the leaning posture that looks like Leaning Tower of Pisa. The exclusion of familial neurological diseases and a comprehensive neurological assessment are crucial in the diagnostic evaluation. Walking and sitting usually worsen the posture of PS. Additionally, patients with PS are indifferent to their abnormal posture. ${ }^{3,5,8,9)}$

Pathophysiology of PS has not been fully understood, but serotonergic or noradrenergic dysfunctions together with dopaminergic-cholinergic imbalance have been suggested. ${ }^{5)}$

This paper aimed to discuss the clinical manifestation of PS in an adolescent with mental retardation who has been receiving risperidone. Informed consent was taken from the patient's parents for publication of this case report.

\section{CASE}

A 15-year-old male patient was admitted to pediatric neurology outpatient clinic for the complaint of abnormal posturing. Developmental history revealed that he had been diagnosed with severe mental retardation in early childhood. He had marked delays in basic motor skills, never begun speaking and has been receiving special education for six years. He attended a child and adolescent psychiatry outpatient clinic four years ago for hyperactivity, irritability, aggressiveness, and self-injurious behavior. With the diagnoses of attention deficit hyperactivity disorder, conduct disorder and mental retardation, he has been on risperidone $2 \mathrm{mg}$ /day for the last four years. Approximately one month ago, the patient gradually de-

(c) This is an Open-Access article distributed under the terms of the Creative Commons Attribution Non-Commercial License (http://creativecommons.org/licenses/by-nc/4.0) which permits unrestricted non-commercial use, distribution, and reproduction in any medium, provided the original work is properly cited. 
veloped tonic flexion of trunk and head toward left. With the suspect of an adverse effect, medical records were carefully reviewed for a detailed medication history. The patient had used no psychotropic medication other than risperidone through these four years. No antiemetic use was also found. Medical records revealed the use of antibiotics and antipyretics 3-4 times a year for colds, upper respiratory tract infections, and bronchitis. No movement and neurodegenerative disorder were reported in family history.

Neurologic examination showed that the patient had clear consciousness, with no abnormalities in sensation of the extremities, cranial nerve functions, muscular strength and deep tendon reflexes. He could not speak, but he could follow the orders. Tonic flexion of trunk and head to the left and shift of the center of gravity toward the left were observed (Fig. 1). He did not have any history of dystonic movements and no evidence of other extrapyramidal symptoms was found. Common blood and biochemical tests were in normal range. Magnetic resonance imaging (MRI) of the brain detected global cortical atrophy, agenesis of the corpus callosum and no abnormality in basal ganglia.

Due to the emergence of abnormal posture after longterm risperidone use, the diagnosis of PS was suspected. Possible neurological diseases were excluded by family history, neurological examination, laboratory tests and neuroimaging. Risperidone dose was decreased to 1 $\mathrm{mg}$ /day based on the recommendation of child and adolescent psychiatry department. Two weeks following the dose reduction, abnormal posture and flexion of the trunk and head disappeared. Hence, the diagnosis of risperidone induced PS has been verified (Fig. 2).

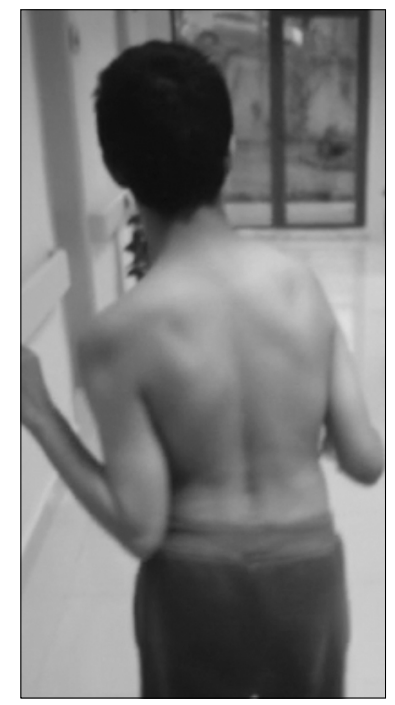

Fig. 1. Tonic flexion of trunk and head to the left.

\section{DISCUSSION}

Among the medication induced extrapyramidal side effects, PS can be defined as a specific type. Dysfunction of cerebral dopaminergic pathways, which regulates axial muscle tone, has been suggested for the pathophysiology of PS. Axial dystonia eventually brings out the typical posture of the syndrome. ${ }^{8)}$ In the present case, with no evidence of any organic etiology, long-term antipsychotic use appears to be the direct cause of PS. To the best of our knowledge, this is the first adolescent case of PS induced by antipsychotic treatment.

In the literature, a number of atypical antipsychotics including clozapine, olanzapine, sertindole, risperidone, aripiprazole and quetiapine have been reported to cause PS. ${ }^{4-8,10-12)}$ Risperidone has a high affinity to dopaminergic $\mathrm{D}_{2}$ receptors. Strong $\mathrm{D}_{2}$ receptor blockade, while being the main mechanism of efficacy, is also responsible for the increased risk of extrapyramidal symptoms (EPS) especially in higher doses. ${ }^{8)}$ In the present case, long-term risperidone use might have caused PS by the prolonged blockade of $\mathrm{D}_{2}$ receptors. The dose of $2 \mathrm{mg}$ /day may be considered as somehow a low dose for EPS risk; however, children have been shown to be more vulnerable to EPS with atypical antipsychotics. ${ }^{13)}$ The presence of mental retardation might also predispose the case to PS. Previous studies have shown that patients with mental retardation experience EPS with lower doses of antipsychotics. ${ }^{14)}$

Children with autism spectrum disorders and mental retardation usually present stereotypic behaviors and motor mannerisms. Some of these compulsive behaviors may al-

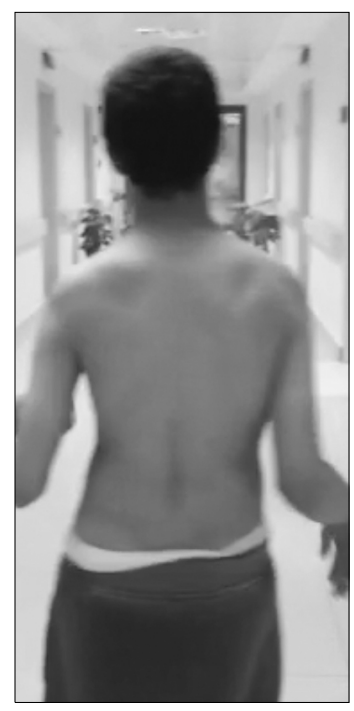

Fig. 2. Two weeks after the dose reduction. 
so be accompanied with an abnormal posture. ${ }^{14,15)}$ Therefore, the posture of PS may be confused with these behaviors by clinicians and may be underdiagnosed. PS should be kept in mind in patients with autism spectrum disorders or mental retardation taking antipsychotic medications.

Besides antipsychotic use, main risk factors for PS include older age, female gender, organic brain injury and combined pharmacological treatment. Among these factors, combined pharmacological treatment has been frequently mentioned in the literature. ${ }^{16)}$ It is interesting that the present case has not been receiving combined pharmacological treatment. Our case had corpus callosum agenesis and global cortical atrophy on brain MRI, which might increase the susceptibility to PS. In the literature, Suzuki et al. ${ }^{711)}$ determined frontal or diffuse cortical atrophy and ventricular dilation on computed tomography in half of the PS patients.

Since the underlying mechanism of PS has not been fully clarified, clinical management is usually performed empirically. The first step in treatment is to discontinue the antipsychotic medication or, for the mild cases, to decrease the dose. Second step is considering to initiate anticholinergic treatment. ${ }^{4,8)}$ In line with the literature, risperidone dose was gradually reduced in the present case and PS disappeared approximately two weeks later without the need for any additional treatment.

This case report emphasizes that PS can also occur in young males that receive long-term antipsychotic medications. The clinical presentation and treatment course in the case may be considered as an example of PS in children and adolescents with mental retardation. Future studies are needed to clarify the exact mechanism of PS and its possible differences with other medication induced EPS symptoms.

\section{acknowledgments}

This article was conducted at the Mersin University Medical Faculty.

\section{REFERENCES}

1. Kelly DV, Béique LC, Bowmer MI. Extrapyramidal symptoms with ritonavir/indinavir plus risperidone. Ann Pharmacother 2002;36:827-830.

2. Kim BN. The clinical use of risperidone in child \& adolescent psychiatry. Clin Psychopharmacol Neurosci 2003;1:178-187.

3. Kaufmann A, Boesch S, Fleischhacker WW, Hofer A Management of a risperidone-induced tardive Pisa syndrome: a case report. J Clin Psychopharmacol 2012;32:418-420.

4. Stübner S, Padberg F, Grohmann R, Hampel H, Hollweg M, Hippius $\mathrm{H}$, et al. Pisa syndrome (pleurothotonus): report of a multicenter drug safety surveillance project. J Clin Psychiatry 2000;61:569-574.

5. Suzuki T, Matsuzaka H. Drug-induced Pisa syndrome (pleurothotonus): epidemiology and management. CNS Drugs 2002;16:165-174.

6. Kwak YT, Han IW, Baik J, Koo MS. Relation between cholinesterase inhibitor and Pisa syndrome. Lancet 2000; $355: 2222$.

7. Suzuki T, Kurita H, Hori T, Sasaki M, Baba A, Shiraishi $\mathrm{H}$, et al. The Pisa syndrome (pleurothotonus) during antidepressant therapy. Biol Psychiatry 1997;41:234-236.

8. Cordeiro Q, Zung S, Vallada H. Pisa syndrome induced by rapid increase and high dosage of risperidone. Arq Neuropsiquiatr 2008;66:896-897.

9. Iuppa CA, Diefenderfer LA. Risperidone-induced Pisa syndrome in MS: resolution with lurasidone and recurrence with Chlorpromazine. Ann Pharmacother 2013;47:1223-1228.

10. Walder A, Greil W, Baumann P. Drug-induced Pisa syndrome under quetiapine. Prog Neuropsychopharmacol Biol Psychiatry 2009;33:1286-1287.

11. Suzuki T, Koizumi J, Moroji T, Sakuma K, Hori M, Hori T. Clinical characteristics of the Pisa syndrome. Acta Psychiatr Scand 1990;82:454-457.

12. Rota E, Bergesio G, Dettoni E, Demicheli CM. Pisa syndrome during aripiprazole treatment: a case report. Prog Neuropsychopharmacol Biol Psychiatry 2007;31:286-287.

13. Jensen PS, Buitelaar J, Pandina GJ, Binder C, Haas M. Management of psychiatric disorders in children and adolescents with atypical antipsychotics: a systematic review of published clinical trials. Eur Child Adolesc Psychiatry 2007:16:104-120.

14. Matson JL, Mahan S. Antipsychotic drug side effects for persons with intellectual disability. Res Dev Disabil 2010;31:1570-1576.

15. Mazzone L, Postorino V, Valeri G, Vicari S. Catatonia in patients with autism: prevalence and management. CNS Drugs 2014;28:205-215.

16. Huang YC, Lee Y, Lin PY. Reversible Pisa syndrome associated with switching from haloperidol to aripiprazole during lithium treatment. Prog Neuropsychopharmacol Biol Psychiatry 2009;33:1284-1285. 